\title{
Recent literature with comments
}

(C) Springer-Verlag 2009

\section{Conservative surgery of uterine adenomyosis}

\section{Masato N, Katsumi T, Yuko A, Hirokazu O, Ryota I Conservative surgical management for diffuse uterine adenomyosis. Fertil Steril 2009 doi:10.1016/j.}

Objective: To evaluate the feasibility and the effect of a conservative modified surgical technique performed at laparotomy in a group of patients with diffuse adenomyosis diagnosed at MRI.

Methods: Retrospective analysis of conservative surgical treatment in 44 women with diffuse adenomyosis diagnosed on pelvic examination by an enlarged uterus and by MRI with a hypertrophy of the anterior and posterior walls with loss of the junctional zone. All women desired strongly to avoid hysterectomy. Mean age of the patients was $37.1 \pm 3.8$ y (range 29-45 y). Dysmenorrhea and abnormal uterine bleeding were the main complaints. An asymmetric longitudinal incision was performed sacrificing one Fallopian tube as it was cut at the interstitial portion. The serosal incision formed a line between the height of the internal os at the anterior/posterior wall and the uterine fundus. From this incision the myometrium was dissected diagonally as if hollowing out the uterine cavity. After transverse opening of the uterine cavity the adenomyosis lesion was excised to a thickness of $5 \mathrm{~mm}$ of the inner myometrium. The lesion was then excised to a thickness of $5 \mathrm{~mm}$ of the serosal myometrium. Consecutively the uterine cavity was closed followed by uterine rejoining with the left side covering the right side.

Results: Histological examinations revealed the presence of adenomyosis in all patients. For dysmenorrhea the mean visual analogue scale decreased from $9.4 \pm 1.0$ before the intervention till $0.8 \pm 1.0$ postoperatively. Postoperative blood loss decreased in all women and anaemia improved in all of them. Mean operation time was $159 \pm 43.7 \mathrm{~min}$. Seven patients required transfusion. No postoperative complications occurred and none of the patients showed an Asherman syndrome. One year after surgery 3 of 32 women had recurrence of dysmenorrhea. One woman has an evolutive pregnancy after IVF treatment.

Conclusion: The surgical procedure provides dramatic relief from dysmenorrhea and improves anaemia. The procedure is indicated in those women wanting a relief of their symptoms while preserving the uterus, but is less indicated in women wanting to become pregnant.

\section{COMMENTARY}

This article describes a modified surgical technique for the treatment of diffuse adenomyosis in women wanting to preserve their uterus. It is a rather invasive technique where the uterine cavity surrounded by some myometrial tissue is hollowed out of the rest of the myometrium. After resection of the adenomyotic lesion only $5 \mathrm{~mm}$ of the inner myometrium is saved and $5 \mathrm{~mm}$ of the serosal myometrium.

The authors seem to have a large experience in the surgical treatment of adenomyosis as they performed conservative surgery in 225 patients with adenomyosis in a two-year time period. The modified intervention was performed in 44 of them.

Diffuse adenomyosis is a common finding in parous women, whereas cystic adenomyosis is probably under diagnosed in adolescents and women in their reproductive age (1).

As such the paper is worthwhile reading as it describes a rather original, although invasive, technique for the treatment of diffuse adenomyosis. It is a description for a more 
systematic and structured removal of the adenomyotic lesion rather than a classical reduction surgery. Such a more structured approach also has been described by another Japanese group using an $\mathrm{H}$ incision technique in 6 patients (2) with more evidence of postoperative relief of pain compared to the previous performed classical reduction surgery.

The first point is about the importance of this procedure as the major indication is the willing of the patient to conserve the uterus in presence of diffuse adenomyosis. The most of the patients are parous or multiparous women without further desire of pregnancy, complaining from dysmenorrhea and heavy menstrual bleeding. As the performed intervention is done by laparotomy with a mean hospitalisation time of 11 days, the surgery can be considered a lot more invasive as a laparoscopic total or subtotal hysterectomy with only a hospitalisation time of \pm 2 days, resulting in a reduction of the costs. In presence of extensive adenomyosis and in absence of further desire for reproduction half or two thirds of the uterus can be removed leaving any normal myometrium. However subtotal hysterectomy is preferable to partial hysterectomy. Even incomplete excision resulted in symptom relief for 3 years (3). In his paper the author is reporting up till now a $10 \%$ recurrence rate of dysmenorrhea, but the follow-up period is still short. The probability of a second intervention by means of a hysterectomy is realistic.

A less invasive medical treatment by means of a levonorgestrel releasing intra uterine device could be the first treatment option. Recent publications were reporting the beneficial effect of this device in patients with adenomyosis with a significant reduction of the grade of dysmenorrhea, blood loss and uterine size and decreased CA-125 (3, 4, 5). Hysteroscopic endometrial resection with or without the use of a levonorgestrel releasing intra uterine device is a valid alternative treatment option $(6,7)$.

The described procedure is not an option in patients still desiring to become pregnant. First one Fallopian tube is sacrificed to be able to perform the intervention; secondly there is no evidence that by such a radical removal of the adenomyotic lesion implantation rates will be increased; third obstetric outcome will be impaired with serious elevated risks for uterine rupture $(3,8)$. Comparison with obstetric outcome after myomectomy is not appropriate. A myoma is growing insight the myometrium with displacement of the myometrium and is well encapsulated. Adenomyosis is infiltrating the normal myometrium and is not well delineated. Excision of the diseased area reduces the myometrial mass. This reduction and the production of uterine scars increase the risk for premature labour and uterine rupture (3). In the described surgical technique most of the myometrial mass is removed, it can be questioned if a further pregnancy is still acceptable and should not be avoided? In patients in their reproductive live, adenomyosis is probably under diagnosed in the "unexplained" infertility group (10). Surgery should be reserved to failures of hormonal suppression with GnRh-a $(3,11)$ or to localised adenomyotic lesions. To date there is no agreement on the most appropriate treatment in patients with adenomyosis and infertility. In case of failure of hormonal suppression with GnRh-a, surgery could be an option in well-selected patients. This surgery should be performed following the principles of microsurgery with meticulous haemostasis and continuous irrigation and avoiding opening of the uterine cavity.

In conclusion: although the technique described by the authors result in a significant improvement of the symptoms, follow-up period is short and surgery rather invasive. Indication is questionable. In absence of further desire for children less invasive therapy should be preferred like hormonal suppression, hysteroscopic endometrial ablation or use of a levonorgestrel releasing intra uterine device.

In patients willing to become pregnant this surgery is certainly not an option: there is no evidence that it will improve probability of conception, one Fallopian tube is sacrificed, there is the risk of postoperative adhesion formation and there is a removal of a urge part of the myometrium with an elevated risk for uterine rupture and premature delivery. This surgery should be considered dangerous in patients willing to conceive and it is persiflage on all the principles of microsurgery! His place within the area of reproductive surgery is at least questionable.

\section{Stephan Gordts, Leuven, Belgium}

\section{References}

1. Ball E, Ganji M, Janik G, Koh C (2009) Laparoscopic resection of cystic adenomyosis in a teenager with arcuate uterus. Gynecol Surg, 6:367-70

2. Fujishita A, Masuzaki H, KhanKN, Kitajima M, Ishimaru $\mathrm{T}$ (2004) Modified reduction surgery for adenomyosis. A preliminary report of transvers $\mathrm{H}$ incision technique. Gynecol Obstet Invest 57: 132-8.

3. Wood C (1998) Surgical and medical treatment of adenomyosis Hum Reprod Update 4: 323-336.

4. Sheng J, Zhang WY, Zhang JP, Lu D (2009) The LNGIUS study on adenomyosis: a 3 year follow-up study on the efficacy and side effects of the use of levonorgestel intrauterine system for the treatment of dysmenorrhea associated with adenomyosis. Contraception 79: 189-93.

5. Cho S, Nam A, Kim H, Chay D, Park K, Cho DJ, Park Y, Lee B (2008) Clinical effects of the levonorgestrelreleasing intrauterine device in patients with adenomyosis; Am J Obstet Gynecol 198: 373.e 1-7. 
6. Bragheto AM, Caserta N, Bahamondes L, Petta CA (2007) Effectiveness of the levonorgestrel-releasing intrauterine system in the treatment of adenomyosis diagnosed and monitored by magnetic resonance imaging. Contraception 76: 195-9.

7. Maia H Jr, Maltez A, Coelho G, Athayde C, Coutinho EM (2003) Insertion of mirena after endometrial resection in patients with adenomyosis. J Am Assoc Gynecol Laparosc 10: 512-6.

8. Mabrouk Villa G, Guerrini M, Mignemi G, Colleoni GG, Venturoli S, Seracchioli R. (2008) Uterine rupture in a primigravida with adenomyosis recently subjected to laparoscopic resection of Rectovaginal endometriosis: case report. J Minim Invasive Gynecol 15: 360-1.

9. Heinonen PK, Helin R (2009) Endometrial resection following levonorgestrel intra uterine system treatment for menorrhagia. Gynecol Surg 6: 245-249.

10. Peng-Hui Wang, Tzay-Shing Yang, Wen-Ling Lee, Hsiang-Tai Chao, Sheng-Ping Chang, Chiou-Chung Yuan (2000) Treatment of infertile women with adenomyosis with a conservative microsurgical technique and a gonadotropin-releasing hormone agonist. Fertil Steril 5: 1061-62.

11. Ching-Jung Wang, Leung-To Yuen, Shuenn-Dyh Chang, Chyi-Long lee, Yung-Kuei Soong (2006) Use of laparoscopic cytoreductive surgery to treat infertile women with localized adenomyosis. Fertil Steril 86: $462 \mathrm{e} 5$.

\section{Nerve fibers and pelvic pain-related disorders}

\section{Xinmei Z, Bangchun L, Xiufeng H, Hong X, Caiyun Z, Jun L \\ Endometrial nerve fibers in women with endometriosis, adenomyosis, and uterine fibroids. Fertil Steril 2009;92:1799-1801.}

Objective: To determine whether nerve fibers in the functional layer endometrium are caused by an endometriosis itself or a common symptom of pain.

Methods: Endometrial tissues from 30 women with endometriosis, 40 women with adenomyosis, 41 women with uterine fibroids, and 47 endometriosis women with adenomyosis were stained immunohistochemically using the highly specific polyclonal rabbit antiprotein gene product 9.5 (PGP9.5) and monoclonal mouse antineurofilament protein.

Results: We demonstrated PGP9.5-immunoactive nerve fibers in the functional layer of endometrium in women with pain symptoms, but not in women without pain symptoms, whether the women had endometriosis, adenomyosis, uterine fibroids, or endometriosis with adenomyosis.
Conclusion: The results suggest a role of PGP9.5-immunoactive nerve fibers in the functional layer of the endometrium playing in pain generation in these disorders.

\section{COMMENTARY}

Approximately $15 \%$ of women are affected with chronic pelvic pain. The pain could be related to gynecologic conditions including endometriosis, adenomyosis or uterine fibroids and to non- gynecologic disorders such irritable bowel syndrome or interstitial cystitis. The most widely known cause of chronic pelvic pain is endometriosis and that could be found in $30 \%$ of these women. The severity of endometriosis however is not correlated with the amount of pain. In fact, the origin of the pain remains unclear. Accordingly, studies evaluating the origin of pelvic pain are always welcome.

Several years ago, we reported the presence of nerve fibers in intra-abdominal adhesions, but their presence was not related to the underlying pathology or pelvic pain. In a subsequent study, we found that the presence of nerve fibers in the peritoneum was not related to endometriosis. We then used immunocytochemistry staining with a monoclonal antibody to neurofilament.

Using immunohistochemical staining with highly specific polyclonal rabbit antiprotein gene product 9.5 (PGP9.5), Zhang et al confirmed previous reports that PGP9.5 immunoreactive nerve fibers could be found in functional endometrium of women with pelvic pain independent of the underlying conditions including endometriosis, adenomyosis or uterine fibroid. They postulated that these nerve fibers play a role in the origin of pain related to those conditions. By studying the endometrial tissue, it appears that the authors believe that endometrial innervation is a marker for endometriosis or other pelvic pain- related disorders. It would be useful to evaluate nerve fibers in the peritoneum of women with endometriosis, in the "ectopic endometrial tissue" in women with adenomyosis, and in the myoma and adjacent tissue of those with uterine fibroids. It is unlikely that evaluating the endometrium would explain the source of the pain.

Whether different types of "nerve staining" would produce the same results remains unclear. It is possible that some nerve fibers and nerve endings could not be detected with the current staining methods. Instead of focusing on the presence of the nerve fibers or nerve endings, one should also evaluate the nerve growth factors and their expression in pain-related endometriosis. Confirming their association would allow treatment with "antineurotrophin".

Pain is a subjective symptom. Instead of just using a visual analogue scale, one should consider a more precise pain measurement such as the McGill pain 
questionnaire. Future studies should include women with no gynecologic disorders with and without chronic pelvic pain.

Togas Tulandi, McGill University, Montreal, Canada

\section{Robotic-assisted laparoscopic myomectomy}

\section{Nezhat C., Lavie O., Hsu S., Watson J., Barnett O.,} Lemyre $M$.

Robotic-assisted laparoscopic myomectomy compared with standard laparoscopic myomectomy-a retrospective matched control study. Fertil Steril 2009;91:556-9.

Objective: Compare robotic-assisted laparoscopic myomectomy (RALM) to a matched control standard laparoscopic myomectomy (LM).

Methods: A retrospective matched control study of premenopausal and postmenopausal women who underwent either robotic-assisted or standard laparoscopic myomectomy. Myomectomies were compared with a matched control group of standard LM. Comparisons were based on Fisher's exact, Mann-Whitney, and exact chi-square tests.

Results: Between January 2006 and August 2007, 15 consecutive RALMs were performed at our institution, compared with 35 matched control standard LMs. The two groups were matched by age, body mass index, parity, previous abdominopelvic surgery, size, number, and location of myomas. Mean surgical time for the RALM was 234 minutes (range 140-445) compared with 203 minutes (range 95-330) for standard LMs. Blood loss, hospitalization time, and postoperative complications were not significantly different.

Conclusions: The RALM required a significant prolonged surgical time over LM. It appears that in the hands of a skilled laparoscopic surgeon, the RALM does not offer any major advantage. This technology, however, offers exciting potential applications while learning endoscopic surgery. Further studies are warranted to asses the utility of RALM for general gynecologic surgeons.

\section{COMMENTARY}

This article compares retrospectively robotic assisted and standard laparoscopic myomectomy.

The article concludes that robotic assisted laparoscopic myomectomy (RALM) does not offer any major advantage when compared to laparoscopic myomectomy. Also, the article states that with RALM requires a significant surgical time. We think also from our experience, that total operative time that includes preparation time and surgical time will be also longer with RALM. The authors reported this issue in the results section. However, we think that in other gynaecological surgeries, ie, oncological surgeries, tubal reversal, ... this tool has to be studied.

Another conclusion was added is, that the robotic technology offers a good learning in endoscopic surgery. We noted that for tubal reversal and total hysterectomy that we perform in our department, the learning curve is very short. So, we agree with the authors. However we think that robot training will help the trainees to acquire psychomotor skills and instrument manipulation with the robot, but not laparoscopic instrument manipulation which is totally different, since the interface of each endoscopic approach is different.

The article is a must read. For the moment, in gynaecology, the data that was published speaks more about feasibility of robotic assisted surgery in gynaecology or give series description. We encourage comparative studies, which would be better prospective in the use of robotic surgery in gynaecology.

We do not recommend any change in the surgical practice for myomectomy in light of the results of this article.

Arnaud Wattiez and Joseph Nassif, Strasbourg, France

\section{A benchmark against which to evaluate individual performance}

Harris ID, Styer AK, Petrozza JC.

Ultrasonographer experience does not impact outcomes following ultrasound-guided embryo transfer. Fertil Steril 2009;92:918-22.

Objective: To determine the effect of sonographer experience during ultrasound guided embryo transfer on pregnancy outcomes.

Methods: A total of 319 women who underwent in vitro fertilization and embryo transfer (IVF-ET) from the sitespecific clinic. A total of 118 women (37\%) underwent embryo transfers with a medical assistant (inexperienced ultrasonographer) performing ultrasound guidance, and 201 women (63\%) underwent embryo transfers by an REI fellow (experienced ultrasonographer) performing the US guidance.

Results: The two groups were similar in baseline characteristics or treatment response. Pregnancy outcomes were similar in both groups, with the MA cohort demonstrating clinical pregnancy rate of $43.2 \%$ and a live birth rate of $35.6 \%$, and the REI fellow group yielding a clinical pregnancy rate of $44.8 \%$ and a live birth rate $35.8 \%$, respectively. 
Conclusion: The clinical experience of the person performing ultrasound guidance during IVF-ET does not have an effect on clinical outcome. Thus, the use of an assistant without formal ultrasound training during IVF-ET is a reasonable option.

\section{COMMENTARY}

The main variables that affect nidation are related to uterine receptivity, embryo quality and the efficiency of embryo transfer. This is a landmark publication that assures us that if you have standardised ultrasound technology \& SOPs in place, ultrasonographer experience is not a significant variable. Embryo transfer is the critical step in assisted reproductive technology; with rigid catheters, contamination with blood, mucus or bacteria, increased contraction waves of the myometrium, and the level of difficulty in introducing the catheter inside the uterine cavity tending to reduce embryo implantation rates(1). Searching on Medline revealed that the number of scientific publications on human IVF from the years $1978-2008$ is 50,700 . However, the number of scientific publications on the technique of embryo transfer is only 65 . That discrepancy reflects how little attention has been given to the technique of embryo transfer. It is estimated that poor embryo transfer technique may account for as much as $30 \%$ of all failures in assisted reproduction. Unfortunately, this failure must have affected thousands of couples every year since the beginning of IVF. This final step in assisted reproduction will determine the fate of a long period and a lot of effort, from ovulation induction and ovum retrieval, to the tedious high technology procedures in the laboratory. The probable reason is the apparent simplicity of this maneuver, since most clinicians do not consider inserting a catheter through the uterine cervix and ejecting embryos a difficult task. This facility, however, is relative. Differences in individual transfer performances are reflected in the results reported in the literature. Meldrum et $\mathrm{al}(2)$ and Naaktgeboren et al(3) emphasized that meticulous embryo transfer is essential for the success of IVF. Many services use the 'sensitivity' of the clinician to place the embryos within the uterine cavity at a point close to the fundus(4) similar to the description published by Edwards more than 20 years ago. With respect to this type of embryo transfer, which is more traditional, no attempt has been made to document the variables that might have a negative impact and cause low pregnancy rates and failure of the whole process, such as inadvertent touch of the catheter tip on the fundal endometrial surface or inappropriate embryo placement in the uterine cavity(5). Ultrasonographic observation has many potential advantages: it prevents touching the fundus of the uterus, it confirms that the catheter is beyond the internal os and it permits guidance of the catheter along the endometrial line, a fact that facilitates the use of more flexible catheters. In addition, the full bladder required for transabdominal ultrasound itself is useful for the correction of uterine access through the cervical route in cases of pronounced anteversion-anteflexion. The technique of embryo transfer is very crucial and great attention and time should be given to this step. In order to optimize the embryo transfer technique, several precautions should be taken. The first and most important is to avoid the initiation of uterine contractility. This can be achieved by the use of soft catheters, gentle manipulation and by avoiding touching the fundus. Secondly, proper evaluation of the uterine cavity and utero-cervical angulation is very important, and can be achieved by performing dummy embryo transfer and by ultrasound evaluation of the utero-cervical angulation and uterine cavity length. Ultrasonographic guidance during embryo transfer offers the benefits of catheter visualization to confirm passage beyond the internal os and avoid touching the uterine fundus. In addition, the lack of consistency between uterine position at mock and actual embryo transfer for patients with RV uteri further supports the use of transabdominal ultrasound guidance in order to more accurately assess the cervico-uterine angle at the time of embryo transfer and gently guide the catheter into the endometrial cavity.Both the patient and her partner have the opportunity to be involved and directly visualize the transfer of their embryos to the uterine cavity with USG guidance. It allows their involvement and commentary on the process and the psychological security of the satisfactory completion of the technical components of their treatment cycle.

Using the ovum donation model to eliminate confounding variables, Lindheim et al assessed the impact of US guided ET on pregnancy rates, implantation rates, and multiple gestation rates(6). The authors concluded that US guided ET is simple and reassuring and appears to significantly improve pregnancy outcomes in ovum donation cycles by optimizing the placement of embryos(6). Sallam and Sadek conducted a meta-analysis of randomized controlled studies to evaluate abdominal ultrasound-guided embryo transfer compared to the clinical touch method(7). Out of a total of 2,051 patients: 1,024 received ultrasoundguided embryo transfers and 1,027 received clinical touch method transfers. The authors observed that compared to the clinical touch method, abdominal ultrasound-guided transfer significantly increased the clinical pregnancy rate and the ongoing pregnancy rate. There was no effect on the incidence of ectopic pregnancy, multiple pregnancy, or miscarriage rate(7). Buckett performed a systematic review and meta-analysis of randomized, controlled trials comparing ultrasound-guided embryo transfer with embryo transfer by clinical touch alone(8). Meta-analysis demonstrated a significantly increased chance of clinical pregnancy fol- 
lowing ultrasound-guided embryo transfer in all studies and in the genuinely randomized subgroup. The embryo implantation rate was also significantly increased following ultrasound-guided embryo transfer(8). Ultrasound-guided transfers are the way forward in the next decade \& this paper assures us that if standardised scanning techniques are established in an ART unit, the operator experience or qualification really does not count as a variable affecting ART success rates.

To conclude, ultrasound-guided embryo transfer is definitely useful for improving success rates and ensures the exact position of the catheter in the uterine cavity (and, consequently, the site where the embryos will be transferred and probably implant), in addition to preventing touching the fundal area and thus the occurrence of bleeding and uterine contractions(9). The establishment of a benchmark against which to evaluate individual performance and participation in 'refresher courses' if indicated will help to maximize the implantation rates of the assisted reproductive technology programme as a whole.

\section{Gautam Allahbadia, Mumbai, India}

\section{References}

1. Coroleu B, Carreras O, Veiga A, Martell A, Martinez F, Belil I and Barri P. Embryo transfer under ultrasound guidance improves pregnancy rates after in-vitro fertilization. Hum Reprod 2000;5:616-620.
2. Meldrum DR, Chetkowski R, Steingold KA, de Ziegler D, Cedars MI and Hamilton M . Evolution of a highly successful in vitro fertilization embryo transfer program. Fertil Steril 1987: 64: 382-389.

3. Naaktgeboren N, Broers FC, Heijnsbroek I, Oudshoorn E, Verburg H and Van der Westerlaken L. Hard to believe hardly discussed, nevertheless very important for the IVF/ICSI results: embryo transfer techniquecan double or halve the pregnancy rate. Hum Reprod 1997:12 (Abstract Book 1);149.

4. Schoolcraft WB, Surrey ES and Gardner DK . Embryo transfer: techniques and variables affecting success. Fertil Steril 2001:76;863-870.

5. Mansour RT and Aboulghar MA . Optimizing the embryo transfer technique. Hum Reprod 2002:17;1149-1153.

6. Lindheim SR, Cohen MA and Sauer MV . Ultrasound guided embryo transfer significantly improves pregnancy rates in women undergoing oocyte donation. Int $\mathrm{J}$ Gynaecol Obstet 1999;66:281-284.

7. Sallam HN and Sadek SS . Ultrasound-guided embryo transfer: a metaanalysis of randomized controlled trials. Fertil Steril 2003:80;1042-1046.

8. Buckett WM .A meta-analysis of ultrasound-guided versus clinical touch embryo transfer. Fertil Steril 2003;80:1037-1041.

9. Allahbadia GN, Kadam K, Gandhi G, Arora S, Valliappan JB, Joshi A, Allahbadia S, Wolman I.Embryo transfer using the SureView catheter-beacon in the womb. Fertil Steril. 2009 Mar 5. [Epub ahead of print] 